\title{
Cancer du rectum en 2012
}

\section{Introduction}

\author{
J. Lefevre $\cdot$ Y. Panis \\ (C) Springer-Verlag France 2012
}

Les recommandations pour la pratique clinique n'ont même pas cinq ans et il nous semblait important de proposer un dossier thématique sur le cancer du rectum... Il s'agit en effet d'un exemple typique d'un cancer à la prise en charge réellement multi-disciplinaire, et pour laquelle toutes les étapes ont profondément évolué ces dernières années. Il suffit pour s'en convaincre d'un rapide coup d'œil sur Pubmed : 2256 articles publiés en 2010 et 2273 en 2011 soit plus de 6 articles quotidiens...

Les grandes études ont confirmé l'intérêt du traitement néoadjuvant, de l'exérèse optimale du mésorectum, des possibilités de résection inter-sphinctérienne, et de la place de plus en plus importante de la laparoscopie... mais plusieurs tendances de recherche se dégagent depuis quelques années :

- Plus de conservation sphinctérienne avec l'aide du traitement néoadjuvant, des nouvelles techniques de résection, des nouvelles marges distales... Le temps de l'amputation systématique pour une tumeur palpée lors du toucher rectal est bien loin,

- Vers des protocoles conservant le rectum ? grâce aux progrès et à l'efficacité de la radio-chimiothérapie, 1 patient sur 5 voit sa tumeur complètement stérilisée ouvrant la voie à de multiples questions : comment augmenter ce

taux de réponse complète et comment l'évaluer ? Que faire en cas de réponse : une simple surveillance, une exérèse locale ou une résection antérieure ?

- De plus en plus de laparoscopie : déjà validée par plusieurs essais et méta-analyses dans le cancer du côlon, elle prend progressivement sa place dans le cancer du rectum, avec des bénéfices postopératoires et des résultats à long terme équivalents à la laparotomie,

- La place centrale de la qualité de vie dans les indications thérapeutiques : ce qui amène à repenser la philosophie du traitement, plus uniquement tourné vers le résultat carcinologique (réduire le taux d'amputation abdomino-périnéale, limiter le nombre de patients irradiés et donc mieux les sélectionner à l'aide notamment de l'imagerie, etc),

- Un meilleur traitement local des «petits » cancers du rectum : grâce à la transanal endoscopic microsurgery (TEM)

Nous avons donc voulu dans ce dossier thématique poser 10 questions à des experts en chirurgie colorectale sur ces points qui sont les sujets des débats actuels. Comme toujours les points importants, les références essentielles de l'EBM sont, pour chaque sujet, mises en évidence pour que ce dossier thématique soit pratique et utile aux praticiens.

Bonne lecture.

\section{J. Lefevre $(\varangle)$}

Service de chirurgie générale et digestive,

Hôpital Saint-Antoine-Assistance Publique des Hôpitaux de Paris (APHP), Université Paris VI, 184, rue du Fbg St-Antoine, 75012 Paris, France

e-mail : Jeremie.lefevre@sat.aphp.fr

Y. Panis

Service de chirurgie colorectale,

Pôle des Maladies de l'Appareil Digestif (PMAD),

Hôpital Beaujon, 100, bd du Gal Leclerc,

92118 Clichy cedex, France 